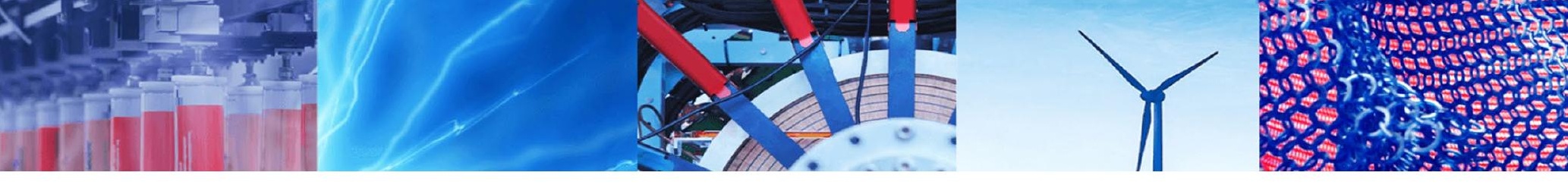

Research Article

\title{
Evolution of microstructures on stainless steel induced by ultra-short pulsed laser ablation
}

\author{
Norbert Ackerl ${ }^{1}\left(\mathbb{D} \cdot\right.$ Gabriela Fisch $^{1} \cdot$ Janko Auerswald $^{2} \cdot$ Konrad Wegener $^{1}$
}

Received: 11 December 2019 / Accepted: 6 March 2020 / Published online: 14 March 2020

(c) Springer Nature Switzerland AG 2020

\begin{abstract}
Ultra-short pulsed laser ablation of stainless steel is accompanied by the evolution of different microstructures. Depending on the fluence, accumulated energy and number of laser passes cones from impurities, laser induced periodic surface structures, cone-like protrusion (CLP), and thermal bumps evolve at the surface. These often unwanted morphologies can be induced or inhibited by carefully choosing the strategy and laser parameters. The investigated range reveals a small processing window for defined $515 \mathrm{~nm}$ sub 1 ps ablation leading to low surface roughness using circular polarization. Hitherto, the origin and dependencies of CLP are still not well understood and for the first time a precursor ripple structure reported. These precursor ripples reveal supra-wavelength periodicity with about $2 \mu \mathrm{m}$ spacing and evolve earliest after the second layer of ablation. Potentially, low spatial frequency laser-induced periodic surface structure generated with the first laser pass with pulse and hatch overlap are the root cause of CLP evolution. Moreover, the CLP growth is grain orientation and strongly polarization state dependent. Preferentially, CLP start to evolve at the $\{110\}$ planes of the face-centered cubic crystals of the inspected austenitic stainless steel and linear polarized laser radiation revealing a 1:1 aspect ratio of $10 \mu \mathrm{m}$. A nanoindentation study at the interface near region on cross-sections reveals robust mechanical properties of this CLP structure.
\end{abstract}

Keywords Laser machining · Self-assembled structures · Cone-like protrusions · Laser induced periodic surface structures · Ultra-short laser pulses · Orthogonal processing $\cdot$ Nanoindentation

\section{Introduction}

In recent years ultra-short pulsed laser machining is becoming a viable technology for industrial application. The equipment costs have been constantly decreasing and several benefits are leading to a strong increase in usage. Having the option to precisely distribute pulses of energy into the material results in a vast number of applications in demand of high precision [1, 2]. Additionally, the high power density allows to ablate and modify otherwise hardto-machine materials like ceramics [3] and diamond [4] as well as synthetic or natural composite materials such as carbon fiber reinforced polymers [5]. Moreover, laser machining can bridge the gap between conventional manufacturing techniques like drilling, milling, electricdischarge machining and semi-conductor technology with the power to span from sub micrometer precision over several meters of geometrical extension.

Laser micromaching of stainless steel is of high interest for such applications as e.g. watch dials, hands, appliques, housings, bracelets or mechanical parts, functionalized surface structures for large scale automotive applications, and corrosion resistant markings in the medical industry [6-8]. However, different microstructures like cones, laser induced periodic surface structures (LIPSS), thermal bumps [9], and cone-like protrusion (CLP) [10] arise

$\triangle$ Norbert Ackerl, ackerln@ethz.ch | 1 Institute of Machine Tools and Manufacturing (IWF), ETH Zurich, Leonhardstrasse 21, 8092 Zurich, Switzerland. ${ }^{2}$ V-ZUG AG, Industriestrasse 66, 6302 Zug, Switzerland. 
depending on the laser parameters and on the optical and physical properties of the target material. In literature slag inclusions from the steel production process have been identified as the origins of cones, which are primarily $\mathrm{Al}_{2} \mathrm{O}_{3}$ and $\mathrm{MgO}$ [11]. In parallel LIPSS of low spatial frequency are generated [12], if linear polarized light is used [13]. Increasing the fluence leads subsequently to the growth of thermal LIPSS, which are perpendicular to the former ones, originating from heat accumulation [14]. Hitherto, there are different regimes of ablation where heat accumulation and power density is the driving force, limiting a high-speed ablation process [15]. However, the CLP structure is not observed after one single layer ablated and, in contrast to thermal bumps, multiple layers of ablation are necessary [16].

Defined ablation conditions are a prerequisite for low roughness and efficient laser ablation [17]. In principle the fluence, pulse-to-pulse distance from laser repetition rate and scan speed leads to a certain overlap linked with the focal beam waist, which are the main parameters [18]. However, depending on the desired process either high material removal rate or surface quality can be reached for $2.5 D$ orthogonal strategies [19] with many layers ablated accumulating stochastic deviations in material removal [20]. This leads to limiting constraints, where high average power must be distributed on the workpiece by high scan speeds and repetition rates [21]. To date there is plenty of laser power with high repetition rates available, but depending on the necessary focal spot size the beam steering [22] or controllers [23] are constraining the maximal laser machining speed. Using the laser in a burst mode with small temporal pulse spacing can enhance the maximal applicable average power [24] and ablation rate [25-27]. However, increasing the number of pulses with sub-nanosecond spaced ultra-short pulses is still a matter of controversy, where the enhanced efficiency for punching could not be observed for multi-pulse $2.5 \mathrm{D}$ strategies [28]. In terms of ablation strategy the laser energy can be distributed on axisymmetric workpieces by a spindle axis without using galvo scanners [29]. Besides orthogonal machining a quasi-tangential irradiation condition under glancing incidence is possible. This allows the use of high average power at fast surface speeds leading to more competitive laser machining processes with decent surface quality and geometric conformity [20,30]. Especially, quasi-tangential laser machining has a huge potential and could utilize the high power nowadays available for ablation, but with increased complexity in the laser path and hatch calculation compared to $2.5 \mathrm{D}$ strategies [31].

Microstructures are not necessarily an unwanted side effect, but can be used to alter the physical interplay at the surface of different materials. Well-known examples are the change in wettability $[32,33]$, optical [34] and, recently, tribological properties [35-37]. The use of laser induced structures for tribology strongly depends on the envisioned application and lubrication condition. The idea proposed in this manuscript is to utilize CLP structures for tribology application with high relative surface speed to reach the hydrodynamic lubrication regime [38-40]. CLP evolve at the surface in a pseudo-periodic arrangement, which can act as reservoir for lubricants helpful to spread a homogeneous film on the surface. A nanoindentation study on cross-sections of these CLP reveals reasonable mechanical properties and cross sections a 1:1 aspect ratio in the regime of several micrometers, potentially applicable to reduce the coefficient of friction.

\section{Experimental techniques}

\subsection{Material}

All studies were carried out on austenitic stainless steel samples of different quality listed in Table 1. The chemical composition is allowed to vary in the given range, where every batch can be slightly different. Steel 1.4310 exhibits a higher carbon content than the widest used austenitic stainless steel 1.4301, resulting in higher mechanical strength desired in certain applications but also in slightly lower corrosion resistance. Steel 1.4404 contains more molybdenum and nickel making it more resistant against acids and saline chloride solutions, e.g. human sweat. For the parameter variation a specimen of $\mathrm{Cr} / \mathrm{Ni}$ stainless steel 1.4301 with $50 \mathrm{~mm}^{2}$ and $2 \mathrm{~mm}$ thickness served as substrate. An initial study on 1.4310 revealed comparable structures and ablation rate, therefore within this contribution not studied in more detail. The ablation characteristic of standard stainless steel sheets was studied on the asreceived state from the supplier without any further sample preparation.

Table 1 Different types of studied stainless steel specimen with the mass percentage of the adjacent species, where iron fills the gap to $100 \%$

\begin{tabular}{llll}
\hline Classification & $\begin{array}{l}1.4310 \\
\text { AISI301 }\end{array}$ & $\begin{array}{l}1.4301 \\
\text { AISI304 }\end{array}$ & $\begin{array}{l}1.4404 \\
\text { AISI316L }\end{array}$ \\
\hline Carbon & $0.05-0.15$ & $\leq 0.07$ & $\leq 0.03$ \\
Chromium & $16.0-19.0$ & $17.0-19.5$ & $16.5-18.5$ \\
Nickel & $6.0-9.5$ & $8.0-10.5$ & $10.0-13.0$ \\
Molybdenum & $0.0-0.8$ & 0 & $2.0-3.0$ \\
Silicon & $\leq 2$ & $\leq 1$ & $\leq 1$ \\
Manganese & $\leq 2$ & $\leq 2$ & $\leq 2$ \\
Grain size & $2.5 \mu \mathrm{m}$ & $8 \mu \mathrm{m}$ & $35 \mu \mathrm{m}$ \\
\hline
\end{tabular}


The small grain size of standard stainless steel materials makes the study of grain dependencies on laser ablation challenging. Therefore, an additive manufactured 1.4404 austenitic steel cube produced by selective laser melting (SLM) has been used. Due to the powder bed fusion technique with the well controlled energy and temperature gradient larger, or similar oriented grain clusters, bigger than $30 \mu \mathrm{m}$, are formed [41]. These grains are elongated in build-up direction, which allows studying the evolution of CLP on the top and side flank of the cube. Here, the specimen was grinded to grit 4000 with $\mathrm{SiC}$ sandpaper and following polished with $\mathrm{Al}_{2} \mathrm{O}_{3}$ suspension with $50 \mathrm{~nm}$ particles for an electron backscatter diffraction (EBSD) measurement. Typical roughness values from optical measurements with a confocal Leica DCM3 microscope following ISO 4288-1996 with a cut-off wavelength of $80 \mu \mathrm{m}$ and a measurement length of $1.5 \mathrm{~mm}$. This points to a arithmetical mean deviation $R_{a}<20 \mathrm{~nm}$ for single profile assessments and a mean area surface roughness $S_{a}<50 \mathrm{~nm}$ with $n>3$ independent measurements for both.

\subsection{Laser machine testbed}

Figure 1a shows the table-top experimental setup constituted by a free path beam delivery and a combination of optical and mechanical axes. An Amphos 200 laser with MOPA configuration and Yb:YAG Innoslab amplification equipped with a second harmonic unit serves as source. The fundamental $1030 \mathrm{~nm}$ has a pulse duration of about 1 ps with a beam quality specified by the supplier of $M^{2}<1.3$, which leads after frequency doubling due to non-linearity to slightly compressed pulses of $0.8 \mathrm{ps}$ at $515 \mathrm{~nm}$ determined by auto-correlation (APE SM1600). The used Amphos laser enables a wide parameter variation in terms of average power up to $120 \mathrm{~W}$ with green light and repetition rates from single shot up to $40 \mathrm{MHz}$. However,

(a)

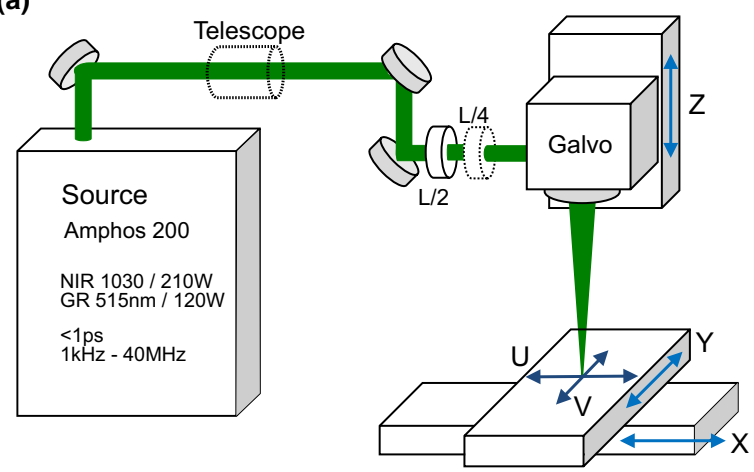

Fig. 1 Laser test bed with mechanical (XYZ) and Galvo (UV) axes for positioning the specimen (a). Pockets with an unablated bridge laser machined with defined polarization state and laser ablation in this study the repetition rate was kept at $800 \mathrm{kHz}$. The laser radiation is coupled into a Scanlab galvo scanner (Hurryscan II), where the raw beam size is controlled by a Galilean telescope (Sill Optics) and polarization set by zero-order wave plates (Altechna). An $163 \mathrm{~mm}$ telecentric f-theta objective lens (Sill Optics) leads to a focal spot diameter of $20 \mu \mathrm{m}$ measured with a beam camera (Ophir Spiricon P620U). Following ISO 11146 , the $D 4 \sigma$ definition is taken being for Gauss-shaped pulses equivalent to the $1 / e^{2}$ describing a reduction in optical intensity to $13.5 \%$. The whole setup is controlled by the Aerotech A3200 software package with axes and controllers from the same supplier. A suction unit with a directional airflow of $250 \mathrm{~m}^{3} \mathrm{~h}^{-1}$ removes and filters the ablation debris.

\subsection{Laser ablation strategy}

For the parameter study pockets are ablated with a $2.5 \mathrm{D}$ strategy and 15 layers, where each adjacent layer is rotated by $23^{\circ}$ mitigating overlap of the hatch lines. The squared pockets have a geometry of $2 \mathrm{~mm}^{2}$ area with a centered unablated region of $1 \mathrm{~mm}$ height and $100 \mu \mathrm{m}$ width, shown in the overview of Fig. 1b. This allows to analyze the total ablated volume, taper flank angle, and precision on the edges of the pockets from the laser machining. Table 2 presents the prospected process parameters with lowest and highest value plus adjacent step size. The average power $P_{\text {avg }}$ leads to a certain fluence $F$ with the focal beam radius $w_{0}$ of $10 \mu \mathrm{m}$, the scan speed $v_{s}$ is equivalent to the pulse-to-pulse distance $d_{\text {pulse }}$ at $800 \mathrm{kHz}$ repetition rate, and the hatch line distance $d_{\text {hatch }}$ is presented with the studied domain.

This leads to a total of 210 laser ablated fields per hatching distance, where the parameters are chosen to cover the evolution of cones, LIPSS, thermal ripples, and partial to full coverage of the pocket surface with CLP structures

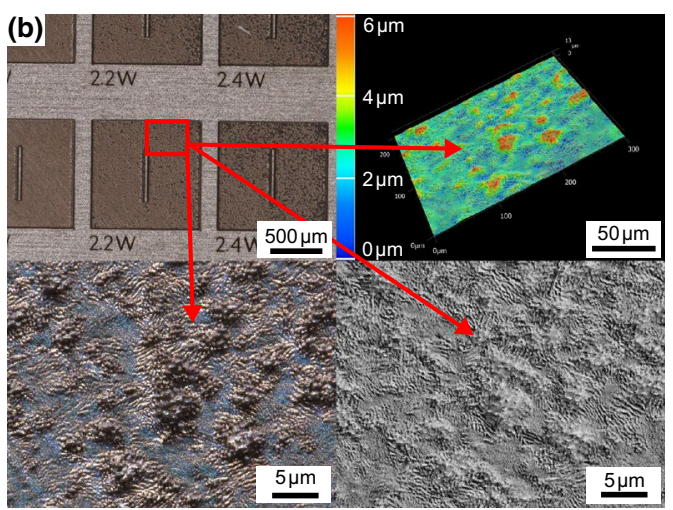

parameters (b). Here, the structure at $1.76 \mathrm{~J} \mathrm{~cm}^{-2}$ and $1.25 \mu \mathrm{m}$ pulseto-pulse distance shows precursor ripples the optical graphs at the bottom 
Table 2 Laser settings and ablation parameters for the study on material removal and evolving microstructures at $800 \mathrm{kHz}$ repetition rate and $20 \mu \mathrm{m}$ focal spot size with the explored values between start and end with step size

\begin{tabular}{llllll}
\hline Range & $\begin{array}{l}P_{\text {avg }} \\
(\mathrm{W})\end{array}$ & $\begin{array}{l}F \\
\left(\mathrm{~J} \mathrm{~cm}^{-2}\right)\end{array}$ & $\begin{array}{l}v_{s} \\
\left(\mathrm{~mm} \mathrm{~s}^{-1}\right)\end{array}$ & $\begin{array}{l}d_{\text {pulse }} \\
(\mu \mathrm{m})\end{array}$ & $\begin{array}{l}d_{\text {hatch }} \\
(\mu \mathrm{m})\end{array}$ \\
\hline start & 0.2 & 0.16 & 100 & 0.125 & 1 \\
end & 2.8 & 2.23 & 1600 & 1.875 & 5 \\
step & 0.2 & 0.16 & 100 & 0.125 & 1 \\
\hline
\end{tabular}

or bumps. Moreover, the dependency of the microstructural evolution on the polarization state is attained by using zero-order half- and quarter-wave plates to adjust and switch the polarization from linear to circular in the focal plane.

The threshold fluence $F_{\mathrm{th}}$, defined by the power density where ablation starts for the multi-pulse regime, of stainless steel was determined for the used samples by considering the assumption for the ablation efficiency [17, 21]. An optimal fluence $F_{\mathrm{opt}}=e^{2} F_{\mathrm{th}}$ follows from a logarithmic ablation law and using a Gauss intensity distribution with a beam waist $w_{0}$ the dependency of the applied peak fluence $F_{0}$ can be studied. The specific material removal, which is the volume removal divided by average power, can be reduced to the ablation depth per layer, if the geometry and pulse-to-pulse distance is kept constant, and follows to

$z_{\mathrm{abl}} / P_{\mathrm{avg}}=\frac{\delta}{2 F_{0}} \ln ^{2} \frac{F_{0}}{F_{\mathrm{th}}}$.

This relation can be used to carry out a least square fitting procedure, where the energy penetration depth $\delta$ and threshold can be attain for a larger set of studied fluence values at one scan speed. This procedure leads to a comparable threshold for the 1.4301 and 1.4404 material between 0.3 and $1.6 \mathrm{~m} \mathrm{~s}^{-1}$ pointing to $(0.065 \pm 0.010) \mathrm{J} \mathrm{cm}^{-2}$ in good agreement with literature for the multi-pulse ablation threshold [21]. Considering single pulse assessments, the saturation after incubation [42] for more than 100 pulses, with the given threshold from the carried out Liu plot analysis [43] reveal a comparable threshold fluence [14, 44].

\subsection{Analysis methods}

The pockets are analyzed per set of parameters with optical focus-shifting and scanning electron microscopy (SEM). By the focus shifting technique of the Keyence VH-X6000 the microstructure is analyzed qualitatively, depicted in Fig. 1 b. As an example of the six shown fields CLP structures can be observed starting at $2.2 \mathrm{~W}$, where the specific process parameters are $1.76 \mathrm{~J} \mathrm{~cm}^{-2}$ fluence, $1.25 \mu \mathrm{m}$ distance between the pulses and $5 \mu \mathrm{m}$ hatch distance. The magnified optical graphs with enhanced contrast show already precursor ripple structures in the vicinity of the CLP. To study the microstructural evolution, the Keyence microscope is implemented on the laser machining testbed and automatically triggered. This allows to take a micrograph after each ablated layer with high resolution. Smaller structures are assessed by SEM using a Hitachi SU-70. The EBSD study was carried out on a FEI Quanta device under $70^{\circ}$ incidence angle of the electron beam. Confocal microscopy using a Keyence VK-X1000 device with a blue laser allows to measure the ablation depth in a sequenced automated manner for all generated pockets. The ablation depth was taken at three line profiles over the unablated centered region in the pocket, compare Fig. $1 \mathrm{~b}$ top left.

To assess the laser-modified region, cross-sections are created by cutting, $\mathrm{SiC}$ grinding with up to grit 4000 and polishing using $\mathrm{Al}_{2} \mathrm{O}_{3}$ particles following the procedure for the preparation of the EBSD SLM specimen. Beside an optical inspection of the interface region, nanoindentation allows to determine the mechanical properties. A Nanomechanics iNano with the continuous dynamic oscillation (CSM) method enables the determination of sub-surface bulk mechanical properties [45]. This technique oscillates the indenter with a sinusoidal driving force, here $110 \mathrm{~Hz}$ and measures force plus displacement with high resolution. The force and indentation depth was calibrated to measure bulk properties and not just the surface near oxide layer [46]. This allowed an indentation distance of $2 \mu \mathrm{m}$, which is a space of more than three times the indentation depth, to avoid the spillover from neighboring indents. The CSM measurement facilitates to calculate the material hardness and elastic modulus for each indent with known Poisson ratio from the adjacent material, revealing the spatial distribution of mechanical properties.

\section{Results and discussion}

\subsection{Microstructural evolution}

Upon the ablation of steel distinct structures are observed and Fig. 2 gives an overview of three characteristic types. Cones, shown in Fig. 2a, appear after ablating several layers near the threshold fluence and are originating from $\mathrm{MgO}$ and $\mathrm{Al}_{2} \mathrm{O}_{3}$ inclusions from the steel production. Due to the higher threshold fluence of ceramics compared to metals, the oxides are ablated less forming cone structure [11]. In parallel LIPSS with low and high spatial frequency (LSFL, HSFL) appear in case of linear polarized laser light. Increasing the energy density leads to the 


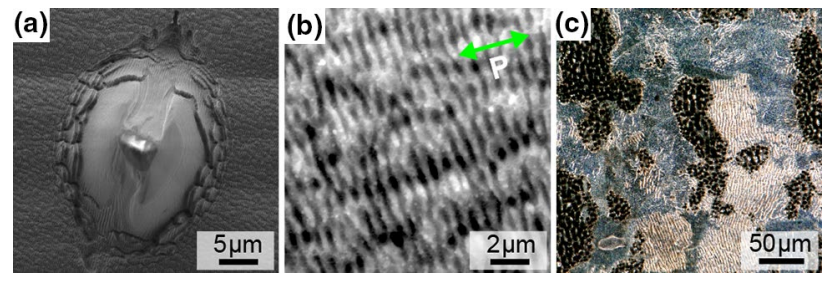

Fig. 2 Inclusions with higher threshold fluence leading to cones (a) and in parallel thermal LIPSS are observed (b) at linear polarization and $0.16 \mathrm{~J} \mathrm{~cm}^{-2}$ fluence. Using circular polarization at a fluence of $2 \mathrm{~J} \mathrm{~cm}^{-2}$ reveals black CLP structure evolving on gray ripple precursor structures (c) after ablating 10 layers

formation of thermal ripples, which can be superimposed to the LIPSS depicted in Fig. 2b. These structural entities are formed in a single pass laser process up to the optimal fluence $F \approx e^{2} F_{\text {th }}=0.48 \mathrm{~J} \mathrm{~cm}^{-2}$ with slow scanning speed and small hatching distance and are well studied in literature [13]. However, at higher fluence starting at $1 \mathrm{~J} \mathrm{~cm}^{-2}$ cone-like protrusions emerge after several layers of ablation, growing with each layer covering a larger portion of the surface observable in the micrograph Fig. $2 \mathrm{c}$ with enhanced optical contrast. The optical graph shows the CLP structure in black and gray ripples on distinct grains of the SLM additively manufactured 1.4404 stainless steel sample. Here, the specimen was etched for $2 \mathrm{~min}$ with $\mathrm{V} 2 \mathrm{~A}$ to reveal the grains and make the preferential growth on certain orientations optically observable. The micrograph presents the surface after ablating 10 layers at $2 \mathrm{~J} \mathrm{~cm}^{-2}$ with a scan speed of $500 \mathrm{~mm} \mathrm{~s}^{-1}(0.625 \mu \mathrm{m}$ spot distance) and $4 \mu \mathrm{m}$ hatch distance. Interestingly, this gray ripple structure seems to serve as precursor to the CLP growth and it is anticipated that the reflectivity and, therefore, energy coupling to the material is altered similar to nanostructures like LIPSS, holes, and bumps [47]. This leads to higher absorbance, which eases surface-near melting, counter-intuitively by using ultra-short pulses of less than $1 \mathrm{ps}$, recently reported to happen at a time scale of subnanosecond [48]. With each subsequent pass of the laser both structures grow laterally and after a certain amount of layers, at a set of laser ablation parameters, the surface is fully covered with CLP.

Inspecting the parameter study from table 2 on standard 1.4301 stainless steel reveals the dependence of the microstructural evolution on fluence and pulse-to-pulse distance after ablating 15 layers with the introduced $2.5 \mathrm{D}$ strategy at $4 \mu \mathrm{m}$ hatch distance in Fig. 3 . In detail, the material removal in terms of one layer is presented and the discussed structures after laser machining pointing to clear growth regimes. The contour plot points to a saturation in terms of material removal rate at increasing fluence with adjacent layer depth $z_{\text {layer }}$, where the slope of the iso-ablation depth lines decreases at about $10 \cdot F_{\text {th }} \approx 0.65 \mathrm{~J} \mathrm{~cm}^{-2}$
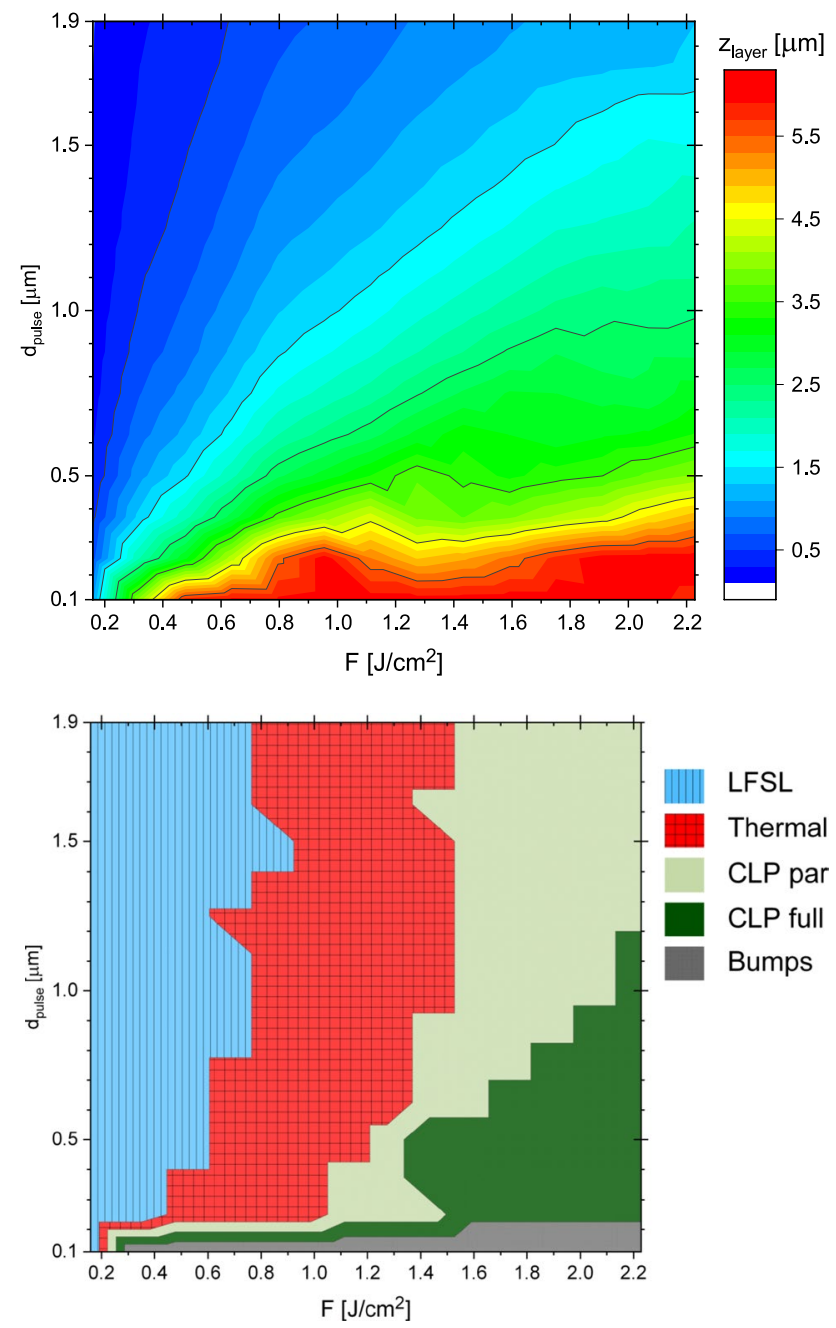

Fig. 3 Layer thickness $z_{\text {layer }}$ and microstructures observed in dependence of fluence and pulse distance at $800 \mathrm{kHz}$ repetition rate after removing 15 layers with linear polarization. A distinct transition from bumps, cones, LIPSS, thermal ripples, and CLP with partial and full area coverage on laser process parameters is revealed

in good agreement with proposed models in literature concerning efficient laser ablation [17, 21]. At low feed rates and small distance between two pulses starting from moderate fluence of $0.3 \mathrm{~J} \mathrm{~cm}^{-2}$, bumps due to heating and melting are formed, where the characteristic semispherical morphology with several $10 \mu \mathrm{m}$ of size covers the surface [49]. Clearly, different microstructures evolve in certain regimes of fluence and pulse-to-pulse distance, where Fig. 3 shows the mapping for $4 \mu \mathrm{m}$ of hatch distance. If the hatching distance is varied, the whole dependency is shifted with comparable tendency and higher total energy input. If now a certain microstructure is desired, a set of parameters can be chosen from this plot or, vice-versa, a certain structure prevented. The LSFL structure can be minimized by the use of circular polarized light, which 
additionally has a strong impact on the precursor ripple formation and following CLP coverage of the surface [50]. However, the larger gray precursor ripple structure evolves in both polarization states with periodicity larger the incident wavelength with about $2 \mu \mathrm{m}$ spacing and convection at the surface molten layer might play a crucial role [51].

\subsection{Impact of grain orientation and polarization}

To further assess the growth dependencies of CLP structures EBSD mapping was carried out on a SLM manufactured polished sample prior laser ablation, see Fig. 4 a.

The inverse pole figure point to the directions of the plain [001], surface diagonal [101] and volume diagonal [111] and the axes of the (001) plane from the facecentered cubic ( $\mathrm{fcc}$ ) crystal structure allow to interpret the received orientation map. Generally, if considering the fcc structure a high symmetry is given and the crystal planes $\{001\}$ and orientations $\langle 101\rangle$ are equivalent. Subsequently, stop-motion optical imaging with the implemented Keyence VH-X6000 microscope mounted on the experimental setup was utilized to observe the growth characteristics of the CLP. From Fig. 3 the laser parameters are selected to $1 \mathrm{~m} \mathrm{~s}^{-1}$ feed rate and $2 \mathrm{~W}$ average power being equivalent to $1.25 \mu \mathrm{m}$ pulseto-pulse distance at $800 \mathrm{kHz}$ repetition rate and a fluence of $1.59 \mathrm{~J} \mathrm{~cm}^{-2}$. This allows to follow the spatial and
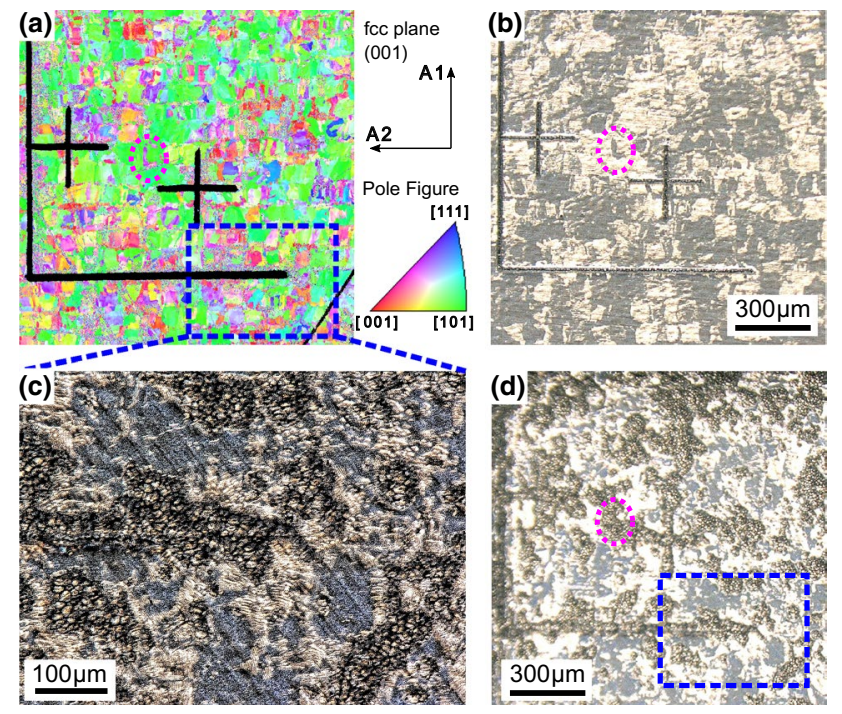

Fig. 4 a EBSD measurement from the pristine polished 1.4404 surface with alignment marks reveal a fcc phase with orientation of the (001) plane and inverse pole figure given. After removing 13 layers with circular polarization at $1.59 \mathrm{~J} \mathrm{~cm}^{-2}$ fluence and $1.25 \mu \mathrm{m}$ pulse-to-pulse distance the grain dependent reflectivity change is apparent (b). With each subsequent layer the CLP evolve preferred on grains oriented in the [101] direction, shown after 80 layers in (c) and the overview in (d)

\section{SN Applied Sciences}

temporal evolution of the microstructures after each ablated layer. Figure $4 \mathrm{~b}$ shows the surface after removing 13 layers by $2.5 \mathrm{D}$ laser machining, where a difference in reflectivity of the adjacent grains is revealed. Clearly, at the $\langle 101\rangle$ orientation directions in the optical micrograph of Fig. $4 \mathrm{~b}$ the reflectivity differs due to surface morphology. These are the proposed precursor ripple structures with a supra-wavelength periodicity shown in Fig. $2 c$ for the SLM specimen and Fig. $1 \mathrm{~b}$ bottom for smaller grains from a standard stainless steel specimen. Following, with each removed layer CLP start to grow randomly on exactly these regions and both structural entities cover larger parts of the surface. In total 80 layers have been removed and the final surface is presented in Fig. $4 \mathrm{~d}$ with a magnification of the blue dashed rectangle in (c). On the graphs a unequivocal preferred growth on the $\{110\}$ planes is observed with a prior reflectivity change. Exemplary, following the magenta ellipse from the EBSD a L-shaped $\{100\}$ grain is embedded in the otherwise green appearing $\{110\}$ planes. The reflectivity of this section keeps darker after 13 layers, and CLP start to evolve on the more shiny regions with layer 14 and subsequently expand laterally on exactly those domains. However, there are some CLP observed on different orientations after 80 layers in Fig. $4 d$, which is dedicated to underlying else-oriented grains appearing after the removal of 80 layers corresponding to $150 \mu \mathrm{m}$ in depth. Generally, the grain clusters are elongated in growth direction induced by the powderbed based SLM process and a cut perpendicular to the build-up direction has been used, confirmed by the determined grain shape from the EBSD measurement. However, the evolution observed layer-by-layer corroborates the strong correlation with grain orientation and the emergence of CLP. Assuming the precursor supra-wavelength ripple structure to be generated by LIPSS and forming thermal ripples due to the fluence well above the threshold, the grain orientation dependency of LSFL could be the origin of the observed grain orientation dependency [52]. However, the preferred growth of CLP at the $\{110\}$ planes presumably could be connected to the packing of the crystal, which changes the coupling with the incident electromagnetic wave. Additionally, a slight change in the thermal conductivity in different orientation of the grains could lead to an accumulation of heat and following thermal ripple formation. Hence, if this is true the growth and appearance should depend on the grain size, which was not observed within this study by comparing the conventional 1.4301 and additive 1.4404 manufactured stainless steel specimen. Nevertheless, this uniquely experimentally attained dependence should provoke a more theoretical assessment in conjunction 

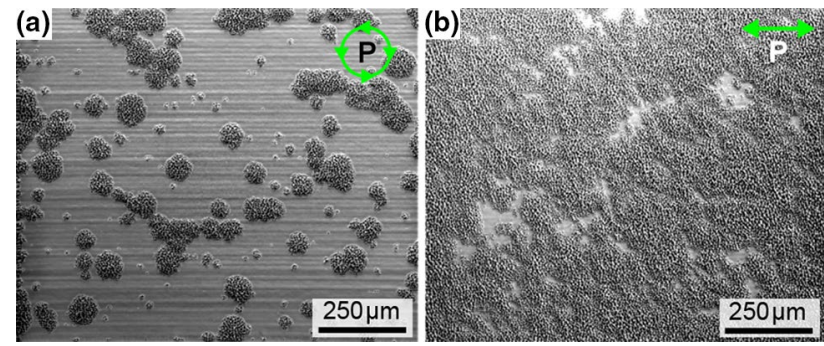

Fig. 5 CLP growth and spatial coverage using circular (a) and linear (b) polarization removing 160 layer at a surface speed of $2.5 \mathrm{~m} \mathrm{~s}^{-1}$ and $3 \mathrm{~J} \mathrm{~cm}^{-2}$

with simulation to unravel the underlying physical mechanism in detail.

In addition to the strong correlation with grain orientation a clear dependence on the laser polarization state is revealed in Fig. 5. A faster set of laser machining parameters with $3.125 \mu \mathrm{m}$ pulse distance at $3 \mathrm{~J} \mathrm{~cm}^{-2}$ is studied. After ablating 160 layer, and keeping the parameters except the polarization state unchanged, the coverage of CLP differs, where circular polarization generates a partial coverage of the surface on several random regions and in contrast linear polarization leads to almost full coverage of the surface shown in Fig. 5a, b accordingly. This strong connection on polarization direction is supposedly correlated with the LSFL formation, which enhances the energy coupling and following thermal ripple creation. A preferred generation of LIPSS starting at defects and discontinuities affecting the surface plasmon polariton, e.g. grain boundaries, could explain the faster growth rate of precursor and consequently CLP. Moreover, even at circular polarization a grain boundary enables LSFL generation matching the defect and polarization direction at certain orientations. This is more unlikely and, therefore, the evolution of LIPSS, ripples and subsequent CLP growth rate is reduced compared to linear polarization. However, this still has to be proven by stop-motion imaging in a SEM to resolve the evolution of the smaller structural units not accessible by optical microscopy. Additionally, a study on the growth rate dependency and evolution of CLP by varying the linear polarization direction on single crystalline specimen could give more insights.

\subsection{Structure and mechanical properties of CLP}

A cross-sectional view on the CLP covered surface in the optical micrographs of Fig. 6 show the evolved protrusions having a semi-periodic pitch of about $10 \mu \mathrm{m}$ and height of $10 \mu \mathrm{m}$. The structure peaks and valleys are higher and deeper than the samples surface, presumably formed by surface-near melting and fast resolidification forming different sized CLP depending on the exact dynamics. Small

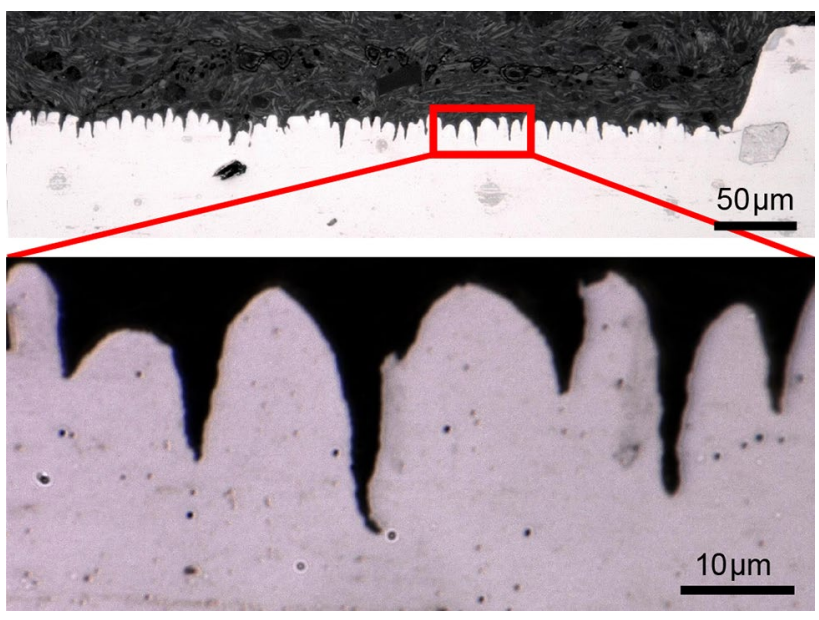

Fig. 6 Cross-section of the CLP structure on 1.4301 stainless steel revealing the size and shape after removing 15 layers with $0.5 \mathrm{~ms}^{-1}$ scan speed at $2 \mathrm{~J} \mathrm{~cm}^{-2}$ energy density and linear polarization

perturbations lead to a variation in height and size of the CLP, compare Fig. 6. A dependence on scanning speed, fluence, and total accumulated energy observed in this study and from literature [10] corroborates this interpretation. The grain size and orientation assessment with EBSD of the introduced CLP structure on 1.4301 steel is still under investigations inhibited by edge charging of the specimen. However, the pseudo-periodic structures appear homogeneously over the whole surface following the dependency on the parameters presented in Fig. 3. This could be taken as an approach for generating microstructures for e.g. changing the wettability and/or enhancing tribological properties for application as discussed in the introduction.

To exploit such microstructures the mechanical stability plays a major role in endurance for the functionality of the surface. Therefore, a nanoindentation study was carried out with an indentation depth of about $100 \mathrm{~nm}$ and a distance of $2 \mu \mathrm{m}$ over a $10000 \mu \mathrm{m}^{2}$ area. Using the CSM method the sample is measured, where the attained stiffness can be used to calculate hardness and E-modulus if the Poisson ratio of the bulk material is known. Near the surface the measured stiffness is slightly reduced, which points to a small change in the mechanical properties of CLP structures, see Fig. 7. However, an impact of the small free standing CLP at the surface, shown in Fig. 6, could interfere with the indentation measurement of the crosssection by a displacement of the whole CLP in indentation direction.

Moreover, a combination of the mechanical properties of the embedding material and the CLP structures could be responsible for the measured decrease at the interface. The calculation for the bulk regime of Fig. 7 with a Poisson ratio $v=0.275$ points to a Young's modulus of $160 \mathrm{GPa}$ in good agreement with literature [53] and at the interface to 


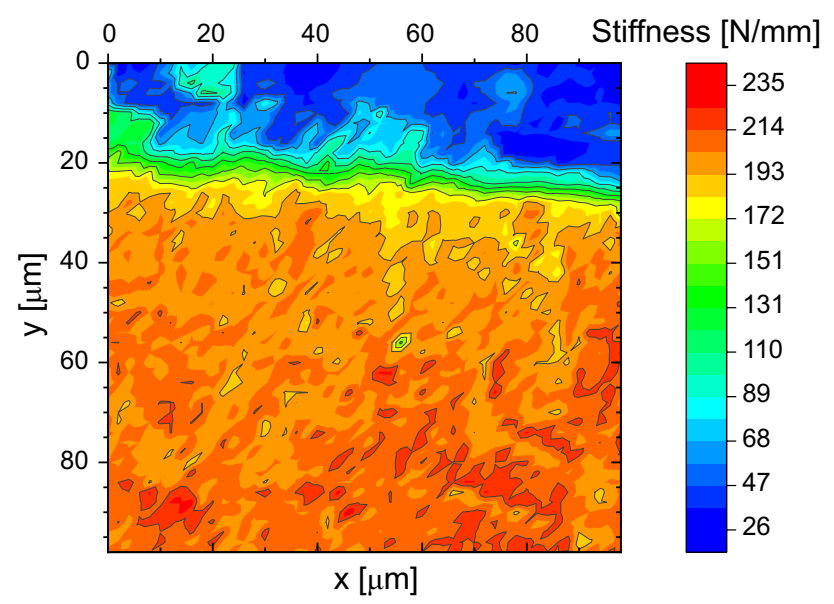

Fig. 7 Nanoindentation with the CSM method showing the measured stiffness. A small change in the surface near region compared to the bulk is revealed. Combined properties of the CLP and embedding material could interfere with the measurement

the embedding material to $120 \mathrm{GPa}$, which corresponds to the green region. Hence, the overall mechanical properties at the surface-near region are good and enable a potential use for tribology. Dimples and microstructures are known to reduce the coefficient of friction for wet lubricant applications with higher surface speeds [39] and LSFL on different substrates have been assessed [37]. It is anticipated, that the CLP could be used in the hydrodynamic regime, where the protrusions act as reservoirs to attain a well spread film. A preliminary experimental study concerning pin on disc measurements with poly-alpha-olefine lubricant gathering the Stribeck curves up to several $\mathrm{m} \mathrm{s}^{-1}$ surface speed revealed a reduction in coefficient of friction from $\mu=0.3$ of the pristine surface to $\mu=0.13$ pointing in the right direction. Nevertheless, a pin diameter with $4 \mathrm{~mm}$ and a normal force of $1 \mathrm{~N}$ was used, which induced fast wear of the microstructures. Generally, tribological experiments demand high standards in terms of sample preparation and surface cleaning prior testing. Surfactants can alter the physical interplay at the solid - liquid boundary changing the coefficient of friction and following wear behavior of the specimen. An envisaged study with increased pin diameter and less force will allow to study the tribological properties in more detail with reduced wear of the CLP structure.

\section{Conclusions}

Ultra-short pulsed laser ablation on stainless steel reveals self-organized microstructures, which depend on the laser and process parameter. The CLP start to grow succeeding at least one ablated layer on ripple precursor structures. With each layer the CLP and ripple structure cover more surface area ending in full coverage of the surface. These two structures preferentially assemble at the $\{110\}$ planes of the face-centered cubic austenitic steel grains under investigation. Moreover, a strong dependence on the laser polarization direction is reported. This is attained to the LSFL evolution, which is depending on grain orientation and boundary defects. A regime for defined ablation without cones and CLP has been identified, where LIPSS can be mitigated by circular polarization. The CLP geometry can be changed by laser parameters, where the resolidification of the surface molten layer plays the crucial role.

A nanoindentation study pointed to robust mechanical properties at the interface compared to the bulk properties with an E-modulus of $160 \mathrm{GPa}$, the outermost boundary layer constituted by CLPs revealed a slight change to about $120 \mathrm{GPa}$. Potentially, these structures are a viable candidate for tribological application to reduce friction and wear via laser surface structuring. A preliminary study pointed to a reduced coefficient of friction in the hydrodynamic regime of lubricated pin-on-disc assessments.

Acknowledgements The authors want to thank the Swiss National Science Fund (FuSSiT/169654) for funding. Thanks to Keyence Switzerland and, especially, Martin Banzer for support and the possibility to implement a Keyence $\mathrm{VH}-\mathrm{X} 6000$ temporarily in our laboratories. Moreover, N.A. wants to acknowledge Reto Völlmin from Keyence Switzerland for support with the confocal Keyence VK-X1000 and setting up the automated measurement routines. The help of Marianna Diamantopoulou with the EBSD studies is highly appreciated. Special thanks from N.A. to Prof. Peter Hosemann at UC Berkeley for many fruitful discussions and hosting during the conception and writing of this manuscript.

\section{Compliance with ethical standards}

Conflicts of interest On behalf of all authors, the corresponding author states that there is no conflict of interest.

\section{References}

1. Chong TC, Hong MH, Shi LP (2010) Laser precision engineering: from microfabrication to nanoprocessing. Laser Photonics Rev 4(1):123. https://doi.org/10.1002/lpor.200810057

2. Malinauskas $M$, Žukauskas A, Hasegawa S, Hayasaki Y, Mizeikis V, Buividas R, Juodkazis S (2016) Ultrafast laser processing of materials: from science to industry. Light Sci Appl 5(8):e16133. https://doi.org/10.1038/lsa.2016.133

3. Ackerl N, Wegener K (2019) Ablation characteristics of alumina and zirconia ceramics on ultra-short pulsed laser machining. J Laser Micro/Nanoeng. https://doi.org/10.2961/ jlmn.2019.02.0009

4. Eberle G, Dold C, Wegener K (2015) Laser fabrication of diamond micro-cutting tool-related geometries using a high-numerical aperture micro-scanning system. Int J Adv Manuf Technol 81 (58):1117. https://doi.org/10.1007/s00170-015-7240-x

5. Freitag C, Wiedenmann M, Negel JP, Loescher A, Onuseit V, Weber R, Abdou Ahmed M, Graf T (2015) High-quality 
processing of CFRP with a 1.1-kW picosecond laser. Appl Phys A 119(4):1237. https://doi.org/10.1007/s00339-015-9159-3

6. Li ZL, Zheng HY, Teh KM, Liu YC, Lim GC, Seng HL, Yakovlev $\mathrm{NL}$ (2009) Analysis of oxide formation induced by UV laser coloration of stainless steel. Appl Surf Sci 256(5):1582. https ://doi.org/10.1016/j.apsusc.2009.09.025

7. Veiko V, Odintsova G, Vlasova E, Andreeva Y, Krivonosov A, Ageev E, Gorbunova E (2017) Laser coloration of titanium films: new development for jewelry and decoration. Opt Laser Technol 93:9. https://doi.org/10.1016/j.optlastec.2017.01.036

8. Garcell EM, Guo C (2019) Colorful multifunctional surfaces produced by femtosecond laser pulses. Opt Mater Express 9(3):1033. https://doi.org/10.1364/ome.9.001033

9. Sedao X, Lenci M, Rudenko A, Faure N, Pascale-Hamri A, Colombier JP, Mauclair C (2019) Influence of pulse repetition rate on morphology and material removal rate of ultrafast laser ablated metallic surfaces. Opt Lasers Eng 116(2018):68. https://doi.org/10.1016/j.optlaseng.2018.12.009

10. Villerius V, Kooiker H, Post J, Pei Y (2019) Ultrashort pulsed laser ablation of stainless steels. Int J Mach Tools Manuf 138(2018):27. https://doi.org/10.1016/j.ijmachtool s.2018.11.003

11. Ling EJY, Saïd J, Brodusch N, Gauvin R, Servio P, Am Kietzig (2015) Investigating and understanding the effects of multiple femtosecond laser scans on the surface topography of stainless steel 304 and titanium. Appl Surf Sci 353:512. https://doi. org/10.1016/j.apsusc.2015.06.137

12. Yao J, Zhang C, Liu H, Dai Q, Wu L, Lan S, Gopal AV, Trofimov VA, Lysak TM (2012) Selective appearance of several laserinduced periodic surface structure patterns on a metal surface using structural colors produced by femtosecond laser pulses. Appl Surf Sci 258(19):7625. https://doi.org/10.1016/j.apsus c.2012.04.105

13. Faas $S$, Bielke $U$, Weber R, Graf T (2018) Prediction of the surface structures resulting from heat accumulation during processing with picosecond laser pulses at the average power of 420 W. Appl Phys A 124(9):612. https://doi.org/10.1007/s0033 9-018-2040-4

14. Mannion P, Magee J, Coyne E, O'Connor G, Glynn T (2004) The effect of damage accumulation behaviour on ablation thresholds and damage morphology in ultrafast laser micro-machining of common metals in air. Appl Surf Sci 233(1-4):275. https ://doi.org/10.1016/j.apsusc.2004.03.229

15. Gillner A, Finger J, Gretzki $P$, Niessen $M$, Bartels $T$, Reininghaus M (2019) High power laser processing with ultrafast and multiparallel beams. J. Laser Micro/Nanoeng. https://doi.org/10.2961/ jlmn.2019.02.0003

16. Ling EJY, Saïd J, Brodusch N, Gauvin R, Servio P, Kietzig AM (2015) Appl Surf Sci 353:512. https://doi.org/10.1016/j.apsus c.2015.06.137

17. Žemaitis A, Gaidys $M$, Brikas M, Gečys P, Račiukaitis G, Gedvilas $M$ (2018) Investigating and understanding the effects of multiple femtosecond laser scans on the surface topography of stainless steel 304 and titanium. Sci Rep 8(1):1. https://doi.org/10.1038/ s41598-018-35604-z

18. Ackerl N, Gysel J, Warhanek MG, Wegener K (2019) Ultrashort pulsed laser manufacturing of yttria stabilized alumina-toughened zirconia dental implants. In: Rechmann $P$, Fried D (eds) Lasers Dent., XXV edn. SPIE, Bellingham, p 18 $10.1117 / 12.2507495$

19. Campanelli S, Ludovico A, Bonserio C, Cavalluzzi P, Cinquepalmi $M$ (2007) Experimental analysis of the laser milling process parameters. J Mater Process Technol 191(1-3):220. https://doi. org/10.1016/j.jmatprotec.2007.03.005

20. Zhou C, Deng H, Chen G (2016) Study on methods of enhancing the quality, efficiency, and accuracy of pulsed laser profiling. Precis Eng 45:143. https://doi.org/10.1016/j.precisione ng.2016.02.005

21. Neuenschwander B, Jäggi B, Schmid M, Hennig G (2014) Surface structuring with ultra-short laser pulses: basics, limitations and needs for high throughput. Phys Proc 56:1047. https://doi. org/10.1016/j.phpro.2014.08.017

22. Jäggi $B$, Neuenschwander B, Zimmermann $M$, Zecherle $M$, Boeckler EW (2016) Time-optimized laser micro machining by using a new high dynamic and high precision galvo scanner. In: Neuenschwander B, Roth S, Grigoropoulos CP, Makimura T (eds) Proc. SPIE LAMOM, vol 973513. International Society for Optics and Photonics, Bellingham, p 11. https://doi. org/10.1117/12.2210791

23. Ackerl N, Boerner P, Wegener K (2019) Toward application of hierarchical structures by ultrashort pulsed laser ablation. J Laser Appl 31(2):022501. https://doi.org/10.2351/1.5096079

24. Jäggi B, Förster DJ, Weber R, Neuenschwander B (2018) Residual heat during laser ablation of metals with bursts of ultra-short pulses. Adv Opt Technol 7(3):175. https://doi.org/10.1515/ aot-2018-0003

25. Jäggi B, Remund S, Zhang Y, Kramer T, Neuenschwander B (2017) Optimizing the specific removal rate with the burst mode under varying conditions. J Laser Micro/Nanoeng 12(3):258. https:// doi.org/10.2961/jlmn.2017.03.0015

26. Gaudiuso C, Giannuzzi G, Volpe A, Lugarà PM, Choquet I, Ancona A (2018) Incubation during laser ablation with bursts of femtosecond pulses with picosecond delays. Opt Express 26(4):3801. https://doi.org/10.1364/OE.26.003801

27. Bonamis G, Mishchick K, Audouard E, Hönninger C, Mottay E, Lopez J, Manek-Hönninger I (2019) High efficiency femtosecond laser ablation with gigahertz level bursts. J Laser Appl 31(2):022205. https://doi.org/10.2351/1.5096087

28. Mur J, Petkovšek R (2019) Near-THz bursts of pulses-governing surface ablation mechanisms for laser material processing. Appl Surf Sci 478(2018):355. https://doi.org/10.1016/j.apsus c.2019.01.182

29. Ackerl N, Warhanek M, Gysel J, Wegener K (2020) Ultra-short pulsed laser conditioning of metallic-bonded diamond grinding tools. Mater Des. https://doi.org/10.1016/j.matdes.2020.108530

30. Warhanek M, Mayr J, Dörig C, Wegener K (2017) Accurate microtool manufacturing by iterative pulsed-laser ablation. Lasers Manuf Mater Process 4(4):193. https://doi.org/10.1007/s4051 6-017-0046-y

31. Ackerl N, Warhanek M, Gysel J, Wegener K (2019) Int J Adv Manuf Technol 103(1-4):1105. https://doi.org/10.1007/s00170-01903540-5

32. Lloyd R, Abdolvand A, Schmidt M, Crouse P, Whitehead D, Liu Z, Li L (2008) Path calculation of 7-axes synchronous quasitangential laser manufacturing. Appl Phys A Mater Sci Process 93(1):117. https://doi.org/10.1007/s00339-008-4646-4

33. Sharma CS, Stamatopoulos C, Suter R, von Rohr PR, Poulikakos D (2018) Rationally 3D-textured copper surfaces for laplace pressure imbalance-induced enhancement in dropwise condensation. ACS Appl Mater Interfaces. https://doi.org/10.1021/acsam i.8b09067

34. Ageev El, Veiko VP, Vlasova EA, Karlagina YY, Krivonosov A, Moskvin MK, Odintsova GV, Pshenichnov VE, Romanov VV, Yatsuk RM (2018) Controlled nanostructures formation on stainless steel by short laser pulses for products protection against falsification. Opt Express 26(2):2117. https://doi.org/10.1364/ OE.26.002117

35. Bonse J, Koter R, Hartelt M, Spaltmann D, Pentzien S, Höhm S, Rosenfeld A, Krüger J (2015) Tribological performance of femtosecond laser-induced periodic surface structures on titanium and a high toughness bearing steel. Appl Surf Sci 336:21. https ://doi.org/10.1016/j.apsusc.2014.08.111 
36. Wang Z, Wang CW, Wang M, Zhao QZ (2016) Manipulation of tribological properties of stainless steel by picosecond laser texturing and quenching. Tribol Int 99:14. https://doi.org/10.1016/j. triboint.2016.03.002

37. Bonse J, Kirner S, Griepentrog M, Spaltmann D, Krüger J (2018) Femtosecond laser texturing of surfaces for tribological applications. Mater (Basel) 11(5):801. https://doi.org/10.3390/ma110 50801

38. Mishra SP, Polycarpou AA (2011) Tribological studies of unpolished laser surface textures under starved lubrication conditions for use in air-conditioning and refrigeration compressors. Tribol Int 44(12):1890. https://doi.org/10.1016/j.triboint.2011.08.005

39. Ripoll MR, Simič R, Brenner J, Podgornik B (2013) Friction and lifetime of laser surface-textured and $\mathrm{MoS}_{2}$-Coated $\mathrm{Ti}_{6} \mathrm{Al}_{4} \mathrm{~V}$ under dry reciprocating sliding. Tribol Lett 51(2):261. https:// doi.org/10.1007/s11249-013-0170-6

40. Lin N, Li D, Zou J, Xie R, Wang Z, Tang B (2018) Surface texturebased surface treatments on $\mathrm{Ti}_{6} \mathrm{Al}_{4} \mathrm{~V}$ titanium alloys for tribological and biological applications: a mini review. Mater (Basel) 11(4):487. https://doi.org/10.3390/ma11040487

41. Heeling T, Wegener $K$ (2018) The effect of multi-beam strategies on selective laser melting of stainless steel 316L. Addit Manuf 22(February):334. https://doi.org/10.1016/j.addma.2018.05.026

42. Sun Z, Lenzner M, Rudolph W (2015) Generic incubation law for laser damage and ablation thresholds. J Appl Phys 10(1063/1):4913282

43. Liu JM (1982) Simple technique for measurements of pulsed Gaussian-beam spot sizes. Opt Lett 7(5):196. https://doi. org/10.1364/OL.7.000196

44. Mustafa H, Mezera M, Matthews D, Römer G (2019) Effect of surface roughness on the ultrashort pulsed laser ablation fluence threshold of zinc and steel. Appl Surf Sci 488(2018):10. https:// doi.org/10.1016/j.apsusc.2019.05.066

45. Li X, Bhushan B (2002) A review of nanoindentation continuous stiffness measurement technique and its applications. Mater Charact 48(1):1 1. https://doi.org/10.1016/S1044-5803(02)00192 $-4$

46. Hosemann P (2018) Small-scale mechanical testing on nuclear materials: bridging the experimental length-scale gap. Scr Mater 143:161. https://doi.org/10.1016/j.scriptamat.2017.04.026

47. Rudenko A, Mauclair C, Garrelie F, Stoian R, Colombier JP (2019) Light absorption by surface nanoholes and nanobumps.
Appl Surf Sci 470(2018):228. https://doi.org/10.1016/j.apsus c.2018.11.111

48. Mo MZ, Chen Z, Li RK, Dunning M, Witte BBL, Baldwin JK, Fletcher LB, Kim JB, Ng A, Redmer R, Reid AH, Shekhar P, Shen $X Z$, Shen $M$, Sokolowski-Tinten $K$, Tsui YY, Wang YQ, Zheng Q, Wang XJ (2018) Heterogeneous to homogeneous melting transition visualized with ultrafast electron diffraction. Science (80-. ) 360(6396):1451. https://doi.org/10.1126/science.aar2058

49. Ahmmed KMT, Ling EJY, Servio P, Kietzig AM (2015) Introducing a new optimization tool for femtosecond laser-induced surface texturing on titanium, stainless steel, aluminum and copper. Opt Lasers Eng 66:258. https://doi.org/10.1016/j.optla seng.2014.09.017

50. Lazzini G, Romoli L, Tantussi F, Fuso F (2018) Nanostructure patterns on stainless-steel upon ultrafast laser ablation with circular polarization. Opt Laser Technol 107:435. https://doi. org/10.1016/j.optlastec.2018.06.023

51. Tsibidis GD, Skoulas E, Papadopoulos A, Stratakis E (2016) Convection roll-driven generation of supra-wavelength periodic surface structures on dielectrics upon irradiation with femtosecond pulsed lasers. Phys Rev B 94(8):081305. https://doi. org/10.1103/PhysRevB.94.081305

52. Römer GR, Huis in't Veld AJ, Meijer J, Groenendijk MN (2009) On the formation of laser induced self-organizing nanostructures. CIRP Ann Manuf Technol 58(1):201. https://doi.org/10.1016/j. cirp.2009.03.068

53. Vitos L, Korzhavyi PA, Johansson B (2002) Elastic property maps of austenitic stainless steels. Phys Rev Lett 88(15):155501. https ://doi.org/10.1103/PhysRevLett.88.155501

Publisher's Note Springer Nature remains neutral with regard to jurisdictional claims in published maps and institutional affiliations. 